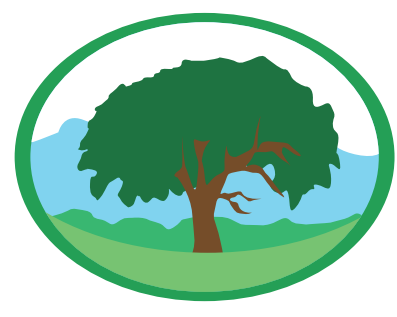

\title{
ESTIMATIVA DA CAPACIDADE EXTRATORA DOS CAPINS VETIVER E TIFTON EM SISTEMAS ALAGADOS CONSTRUÍDOS
}

TEIXEIRA, D. L. ${ }^{*}$; TEIXEIRA, D. V. ${ }^{2}$; MATOS, A. T. ${ }^{3}$; MATOS, M. P. ${ }^{4}$; LEITE JÚNIOR, M. C. R.

Professor adjunto do Instituto de Ciências Agrárias, Universidade Federal dos Vales do Jequitinhonha e Mucuri, Campus Unaí, MG.

${ }^{2}$ Graduanda em Engenharia Agrícola e Ambiental, Universidade Federal dos Vales do Jequitinhonha e Mucuri.

${ }^{3}$ In Memoriam

${ }_{4}^{4}$ Professor adjunto do Departamento de Engenharia Ambiental, Universidade Federal de Lavras, Lavras, MG.

* Avenida Universitária, no 1.000, Bairro Universitários; 38610-000; Brazil. Tel.: +55 (31) 3677-9950; E-mail: denis.teixeiraQuufvjm.edu.br

\begin{abstract}
Palavras-chave: tratamento de água residuária, absorção de nutrientes, modelagem, nitrogênio, fósforo.
\end{abstract}

\section{Resumo}

Neste trabalho, teve-se por objetivo a obtenção de modelos matemáticos para estimativa da capacidade de extração de nitrogênio $(N)$, fósforo $(P)$ e potássio (K) pelo capim-vetiver (Chrysopogon zizanioides) e capim-tifton 85 (Cynodon spp.), quando cultivados em sistemas alagados construídos de escoamento horizontal subsuperficial (SACs-EHSS) cujo meio poroso foi saturado com soluções contendo diferentes concentrações de nutrientes. Os cortes da parte aérea dos capins foram realizados de 30 em 30 dias para determinação da produtividade e dos teores de $\mathrm{N}, \mathrm{P}$ e $\mathrm{K}$ no tecido vegetal. Modelos da capacidade extratora de nutrientes foram obtidos em função da condutividade elétrica (CE) na solução nutritiva. Para validação desses modelos, SACs-EHSS também foram operados em condições de escala plena (SACs-EHSSp), no tratamento de esgoto sanitário. A partir dos resultados obtidos, verificou-se que as extrações de N, P e K pelo capim-vetiver aumentaram linearmente com o aumento da $\mathrm{CE}$ na solução nutritiva. Em relação ao capim-tifton 85, foram ajustados modelos quadráticos de extração de $\mathrm{N}$ e K. Apesar dos modelos ajustados não terem sido capazes de estimar, com exatidão, a variável avaliada, as informações obtidas podem ser empregadas para o aperfeiçoamento dos SACs-EHSSp.

\section{ESTIMATION OF THE EXTRACTION CAPACITY OF VETIVER AND TIFTON GRASSES IN CONSTRUCTED WETLANDS}

\section{Keywords: $\quad$ wastewater} treatment, nutrient absorption, modeling, nitrogen, phosphorus.

\section{Abstract}

The objective of this study was to obtain mathematical models to estimate the capacity of nitrogen $(\mathrm{N})$, phosphorus $(\mathrm{P})$ and potassium $(\mathrm{K})$ extraction by the vetiver grass (Chrysopogon zizanioides) and tifton 85 grass (Cynodon spp.) when cultivated in horizontal subsurface flow constructed wetlands (HSSF-CWs) whose porous medium was saturated with solutions presenting different nutrient concentration. The grass shoots were cut every 30 days to determine the productivity and N, P and $\mathrm{K}$ contents in the plant tissue. Models of the extraction capacity of nutrient were obtained as a function of electrical conductivity $(E C)$ in the nutrient solution. For validation of these models, the HSSF-CWs were also operated under full scale conditions (HSSF-CWsf) for the treatment of sanitary sewage. From the obtained results, it was verified that the extractions of $N, P$ and $K$ by the vetiver grass increased linearly with the increase in EC of the nutritive solution. In relation to tifton 85, quadratic models of $\mathrm{N}$ and $\mathrm{K}$ extraction were adjusted. Although the adjusted models were not able to accurately estimate the evaluated variable, the information obtained can be used to improve the HSSF-CWsf . 


\section{INTRODUÇÃO}

O cultivo de plantas em sistemas alagados construídos de escoamento horizontal subsuperficial (SACs-EHSS) auxilia na remoção de nutrientes da água residuária em tratamento, principalmente, quando estão em pleno desenvolvimento vegetativo e expressam a máxima absorção de nutrientes, além de proporcionar um aspecto agradável.

A absorção de nutrientes pelas plantas é um dos principais mecanismos de extraçáo dos nutrientes dos SACs-EHSS. De acordo com Matos et al. (2009), as plantas utilizam os nutrientes da água residuária para o seu crescimento, atuando como extratoras de grande parte dos nutrientes. Segundo Gao et al. (2014), quanto maior a concentração de nutrientes no efluente em tratamento, maior será a extração pelas plantas, até se atingir um ponto de máximo, que é característico de cada cultura.

A extração e o acúmulo de nutrientes pelas plantas em SACs-EHSS dependem, entre outros fatores, da condutividade elétrica da solução em tratamento, cujos valores são proporcionais à concentração dos vários íons presentes. Portanto, a condutividade elétrica da solução pode afetar a absorção de água e nutrientes, além do acúmulo de biomassa vegetal aérea das plantas cultivadas nos SACs-EHSS (KADLEC; WALLACE, 2008).

No Brasil, existe grande variedade de espécies vegetais com potencial de cultivo em SACs-EHSS, sendo o capim-vetiver (Chrysopogon zizanioides) e o capim-tifton 85 (Cynodon spp.) espécies comumente utilizadas nesses sistemas de tratamento (MATOS et al., 2008, 2009, 2010; DREIFUS, 2012; UCKER et al., 2012; FIA et al., 2015; JESUS, 2016; FIA et al., 2017). O acúmulo de nutrientes na biomassa vegetal aérea é determinado pela produtividade e pela concentração dos nutrientes nos tecidos, sendo o potencial de absorção de nutrientes pelas plantas, geralmente, limitado pela produtividade ou taxa de crescimento, e pelo teor de nutrientes nos tecidos das plantas (GAO et al., 2014).

Considerando-se que o projeto, a operação e a avaliação de SACs-EHSS, possam ser aperfeiçoados, caso se tenha maiores informaçóes em relação à capacidade extratora de nutrientes pelas plantas, neste trabalho teve-se por objetivo a obtenção de modelos de estimativa da capacidade de extração de nutrientes pelos capins vetiver e tifton 85 , em função da concentração de nutrientes, expresso em termos da condutividade elétrica, na solução nutritiva saturante do meio poroso.

\section{MATERIAL E MÉTODOS}

O experimento foi conduzido na Área Experimental de Hidráulica, Irrigação e Drenagem do Departamento de Engenharia Agrícola da Universidade Federal de Viçosa.

\section{Experimento em protótipos de SACs-EHSS para obtenção dos modelos}

A avaliação do efeito da condutividade elétrica (CE) da solução nutritiva saturante do meio poroso na capacidade de extração de nutrientes pelos capins vetiver e tifton 85 , foi realizado a partir do cultivo destas plantas em protótipos de SACs-EHSS, tendo o monitoramento sido feito no período de 5 de março até 17 de novembro de 2016. O experimento foi conduzido em delineamento inteiramente casualizado (DIC), com seis tratamentos (níveis de salinidade) para cada espécie de planta cultivada.

Os protótipos foram construídos em alvenaria, com formato retangular e dimensões médias de $0,92 \mathrm{~m}$ de largura, 0,73 de comprimento e $0,35 \mathrm{~m}$ de altura. $\mathrm{O}$ sistema de drenagem foi confeccionado em tubos de PVC de $32 \mathrm{~mm}$, sendo este colocado em posição oposta ao da entrada da soluçáo nutritiva, mantendose a altura de saturaçáo de $0,25 \mathrm{~m}$. Os protótipos foram preenchidos com uma camada de $0,3 \mathrm{~m}$ de brita "número 0", sendo as mudas do capim-vetiver e do capim-tifton 85 transplantadas com densidade de plantio de 12 propágulos por $\mathrm{m}^{2}$.

Os níveis de salinidade da solução nutritiva saturante do meio poroso foram monitorados considerando-se os valores de CE de 0,2; 0,5; 1,0; 1,5: 2,0 e 3,0 dS $\mathrm{m}^{-1}$. Esses valores foram estabelecidos, tendo como base a faixa de condutividade elétrica comumente encontrada em águas residuárias tratadas em SACsEHSS. O preparo das soluçóes nutritivas era realizado separadamente para cada valor de CE pré-estabelecido, o qual era medido utilizando-se um condutivímetro de bancada, marca Hach, modelo Sension 7. 
$\mathrm{O}$ pH da solução foi mantido entre 5,5 e 6,5, Arnon (1950), sendo as concentraçóes dos nutrientes, utilizando-se soluçôes de ácido clorídrico ou hidróxido para os diferentes valores de CE na solução nutritiva de sódio de $0,1 \mathrm{~mol} \mathrm{~L}^{-1}$. A solução nutritiva utilizada saturante do meio poroso, apresentadas na Tabela 1 . como referência neste trabalho foi a de Hoagland e

Tabela 1. Concentração dos nutrientes para os diferentes valores de condutividade elétrica (CE) na solução nutritiva saturante do meio poroso dos protótipos de SACs-EHSS

\begin{tabular}{|c|c|c|c|c|c|c|c|c|c|c|c|c|}
\hline $\begin{array}{c}\mathrm{CE} \\
(\mathrm{d} S \mathrm{~m}-1)\end{array}$ & -.-. & . & . & $(\mathrm{mg} \mathrm{L}-1)$ & -_-_-_- & - & -.-- & -.- & - & $(\mu \mathrm{g} \mathrm{L}$ & .-...-. & -.- \\
\hline 0,2 & 11,5 & 0,8 & 6,5 & 10,4 & 3,1 & 4,1 & 0,3 & 32 & 1 & 45 & 1 & 6 \\
\hline 0,5 & 38,3 & 3,8 & 24,3 & 25,9 & 7,8 & 10,4 & 0,8 & 81 & 3 & 113 & 2 & 15 \\
\hline 1,0 & 82,8 & 8,9 & 53,9 & 51,8 & 15,6 & 20,7 & 1,6 & 162 & 6 & 227 & 3 & 29 \\
\hline 1,5 & 127,4 & 13,9 & 83,6 & 77,8 & 23,3 & 31,1 & 2,4 & 243 & 10 & 340 & 5 & 44 \\
\hline 2,0 & 172,0 & 19,0 & 113,2 & 103,7 & 31,1 & 41,5 & 3,2 & 324 & 13 & 454 & 6 & 58 \\
\hline 3,0 & 261,1 & 29,0 & 172,4 & 155,5 & 46,7 & 62,2 & 4,9 & 486 & 19 & 680 & 10 & 87 \\
\hline
\end{tabular}

A substituição total da solução nutritiva era realizada de 15 em 15 dias, para evitar prejudicial desbalanceamento dos nutrientes. Já os cortes da parte aérea das plantas foram realizados de 30 em 30 dias, para maximizar a extração de nutrientes pelas plantas, sendo o primeiro corte realizado em 16 de abril e o último em 16 de novembro, totalizando 7 cortes ao longo do período de experimentaçáo.

As análises das plantas foram realizadas no Laboratório de Solos e Resíduos Sólidos do Departamento de Engenharia Agrícola da Universidade Federal de Viçosa, conforme recomendaçóes de Kiehl (1985) e Matos (2015), para análise de tecido vegetal. Para determinação da produtividade de biomassa vegetal aérea, quantificou-se a massa fresca e a massa seca, obtida após secagem em estufa com ventilação forçada a $65^{\circ} \mathrm{C}$, por 48 horas.

A análise nutricional foi realizada em amostras secas, sendo inicialmente trituradas em moinho com rotor de facas e, posteriormente, quantificados os teores de nitrogênio total $(\mathrm{N})$, fósforo $(\mathrm{P})$ e potássio (K). O N foi quantificado de acordo com o processo semi-micro Kjeldahl, enquanto o P e o $\mathrm{K}$ foram quantificados após a digestão nítrico-perclórico das amostras, utilizando-se espectrofotômetro e fotômetro de chama, respectivamente, para quantificação dos teores.

A partir dos dados de produtividade de matéria seca e dos teores de N, P e K nas plantas, foi calculada a capacidade de extração de cada nutriente pelos capins vetiver e tifton 85 (Equação 1).

$$
\text { Ext }_{\text {N,PouK }}=\frac{P \times T_{N_{,} \text {PouK }}}{100}
$$

em que,

Ext. ${ }_{N, P \text { ou } K}$ - extração de N, P ou K pelos capins vetiver ou tifton $85\left(\mathrm{~g} \mathrm{~m}^{-2}\right.$ mês $\left.{ }^{-1}\right)$;

$P$ - produtividade de matéria seca dos capins vetiver ou tifton $85\left(\mathrm{~g} \mathrm{~m}^{-2}\right.$ mês $\left.^{-1}\right)$;

$T_{N \text {, Р ou K }}$ - teor de N, P ou K na matéria seca dos capins vetiver ou tifton $85\left(\mathrm{dag} \mathrm{kg}^{-1}\right)$.

\section{Validaçáo dos modelos obtidos em SACs-EHSS utilizados no tratamento de esgoto sanitário}

Para validação dos modelos desenvolvidos, foram utilizados dados provenientes de dois SACs-EHSS, um cultivado com capim-vetiver e outro com capimtifton 85 , ambos utilizados no tratamento de esgoto sanitário, sistemas que foram denominados, neste trabalho como "escala plena” (SACs-EHSSp).

Os SACs-EHSSp, em operação desde agosto de 2014, foram construídos em paralelo com as seguintes dimensôes: 4,0 $\mathrm{m}$ de comprimento, 1,0 $\mathrm{m}$ de largura e $0,4 \mathrm{~m}$ de profundidade, com fundo nivelado. $\mathrm{O}$ meio suporte é constituído por uma camada de $0,3 \mathrm{~m}$ de brita gnáissica "número 0 ", sendo as mudas dos capins vetiver e tifton 85 transplantadas com densidade de plantio de 12 propágulos por $\mathrm{m}^{2}$. Ao longo dos SACs- 
EHSSp, foram instalados tubos de PVC (40 mm) distanciados a cada $1,0 \mathrm{~m}$ e cravados verticalmente no meio suporte para possibilitar o monitoramento da CE no esgoto sanitário, ao longo do tratamento. Os valores de CE foram utilizados, posteriormente, para validação dos modelos.

Os SACs-EHSSp foram alimentados com uma vazão média igual a $0,22 \mathrm{~m}^{3} \mathrm{~d}^{-1}$, resultando em uma taxa de carregamento orgânico (TCO) de, aproximadamente, $100 \mathrm{~kg} \mathrm{ha}^{-1} \mathrm{~d}-1$ de DBO e tempo de detenção hidráulica de 2,0 dias. Os cortes da parte aérea das plantas foram realizados de 30 em 30 dias, sendo o primeiro corte realizado em 2 de maio e o último em 2 de novembro de 2016, totalizando 7 cortes.

Para validação dos modelos os SACs-EHSSp foram divididos em quatro partes iguais, resultando em uma área de amostragem igual a $1 \mathrm{~m}^{2}$ na qual era determinada a produtividade de biomassa vegetal aérea e a capacidade de extração dos nutrientes, seguindo-se a mesma metodologia descrita anteriormente para os protótipos de SACs-EHSS.

A amostragem do esgoto sanitário afluente aos SACs-EHSSp foi realizada quinzenalmente, durante o período de experimentaçáo, sendo determinados a demanda bioquímica de oxigênio (DBO), nitrogênio total $(\mathrm{N})$, fósforo total $(\mathrm{P})$, potássio total $(\mathrm{K})$ e sódio total $(\mathrm{Na})$ (Tabela 2). As análises laboratoriais foram realizadas no Laboratório de Qualidade da Água do Departamento de Engenharia Agrícola da Universidade Federal de Viçosa, em conformidade com as recomendaçóes do Standard methods for the examination of water and wastewater (APHA, 2012).

Tabela 2. Valores médios de demanda bioquímica de oxigênio (DBO), nitrogênio ( $N$ ), fósforo (P), potássio (K) e sódio ( $\mathrm{Nal}$ no esgoto sanitário afluente aos SACs-EHSSp cultivados com os capins vetiver e tifotn 85, ao longo do período experimental

\begin{tabular}{cccccc}
\hline \multirow{2}{*}{ Datas das Análises } & DBO & N & P & K & Na \\
\hline $02 / 05$ & $211 \pm 19$ & $62 \pm 5,0$ & $7,0 \pm 0,4$ & $7,0 \pm 2,0$ & $34 \pm 3,0$ \\
$02 / 06$ & $212 \pm 48$ & $71 \pm 6,0$ & $8,0 \pm 0,1$ & $11 \pm 2,0$ & $41 \pm 11$ \\
$02 / 07$ & $154 \pm 36$ & $71 \pm 2,0$ & $9,0 \pm 1,0$ & $13 \pm 1,0$ & $47 \pm 6,0$ \\
$02 / 08$ & $151 \pm 39$ & $65 \pm 1,0$ & $7,0 \pm 0,3$ & $12 \pm 2,0$ & $32 \pm 3,0$ \\
$02 / 09$ & $138 \pm 32$ & $82 \pm 11$ & $9,0 \pm 1,0$ & $14 \pm 2,0$ & $45 \pm 4,0$ \\
$02 / 10$ & $156 \pm 12$ & $90 \pm 2,0$ & $9,0 \pm 2,0$ & $11 \pm 1,0$ & $41 \pm 1,0$ \\
$02 / 11$ & $133 \pm 15$ & $76 \pm 1,0$ & $9,0 \pm 1,0$ & $12 \pm 1,0$ & $41 \pm 1,0$ \\
\hline
\end{tabular}

\section{Análises estatísticas}

Foram realizadas análises de regressão para cada planta e para cada corte realizado nos protótipos de SACs-EHSS, obtendo-se 7 modelos de estimativa da capacidade de extração de N, P e K em função da CE na solução nutritiva, sendo considerado satisfatório os ajustes que apresentaram comportamento biológico explicável e significância mínima de $10 \%$ para os coeficientes.

Os parâmetros dos modelos obtidos foram considerados variáveis dependentes e submetidos à análise de agrupamento por otimizaçáo de Tocher, para determinar quais cortes possuíam modelos similares. As análises de regressão e de agrupamento foram realizadas utilizando o programa estatístico $\mathrm{SAEG}^{\circledast}$.

Para validar os modelos provenientes dos protótipos de SACs-EHSS, foram utilizados os valores de CE no esgoto sanitário em tratamento para estimar a extração de $\mathrm{N}, \mathrm{P}$ e K pelas plantas, ao longo do sistema de tratamento. Em seguida, plotaram-se os valores estimados com intervalo de confiança de $95 \%$, conjuntamente com os valores reais obtidos, sendo os resultados submetidos à estatística descritiva para avaliação dos modelos desenvolvidos.

\section{RESULTADOS E DISCUSSÃO}

\section{Modelagem da capacidade de extração de nutrientes dos capins vetiver e tifton 85 cultivados em SACs-EHSS}

$\mathrm{Na}$ Figura 1, estão apresentadas as curvas de capacidade de extração de N, P e K pelos capins vetiver e tifton 85 , em função da CE na solução nutritiva, para 
as 7 datas de corte avaliadas.
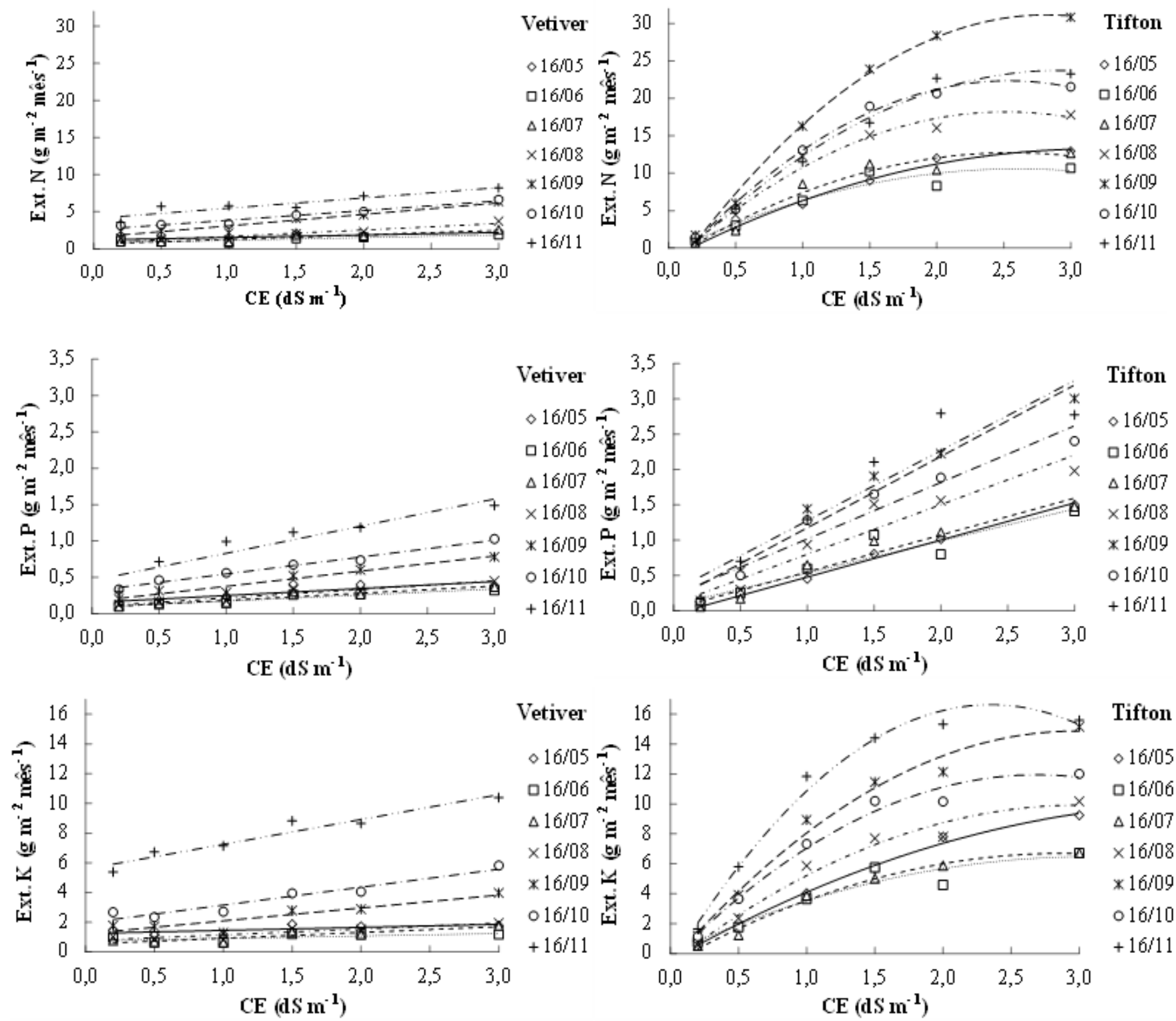

Figura 1. Capacidade de extração de nitrogênio (Ext. N), fósforo (Ext. P) e potássio (Ext. K) pelos capins vetiver e tifton 85, em função da CE na solução nutritiva saturante do meio poroso dos SACs-EHSS.

Considerando os resultados de extração de nutrientes pelas plantas nos SACs-EHSS, verificase que o aumento da concentração de nutrientes, expresso em termos da CE na solução nutritiva, resulta em maiores taxas de extração. De acordo com Musyimi et al. (2010), a maior disponibilidade de nutrientes para as plantas pode aumentar a taxa de absorçáo pelo sistema radicular, favorecendo o acúmulo de nutrientes no tecido vegetal, bem como, o aumento da área foliar para transpiração e fotossíntese.

Observa-se, na Figura 1, que a extração de nutrientes proporcionada pelo capim-vetiver é inferior à do capim-tifton 85 , além disso, a variação na capacidade de extração de $\mathrm{N}$ e $\mathrm{K}$ em função da $\mathrm{CE}$ na solução nutritiva apresenta comportamento linear, no que se refere ao capim-vetiver, e quadrático, para o capim-tifton 85. Isso demonstra a maior capacidade do capim-tifton 85 em acumular nitrogênio no tecido vegetal, característica importante para seleção de plantas a serem utilizadas em SACs-EHSS, onde se deseja maior capacidade de extração de nitrogênio do afluente a ser tratado.

A pequena variabilidade encontrada na extração de $K$ pelo capim-vetiver com a variação da CE na solução nutritiva, demonstra que a extraçáo de $\mathrm{K}$ pela parte aérea das plantas, ao longo comprimento dos SACs- 
EHSS, pode ser considerada constante, corroborando os resultados obtidos por Jesus (2016), no qual não se observou diferença significativa na extração de K pelo capim-vetiver nas diferentes posições de cultivo nos SACs-EHSS.

Em relação à extração de $\mathrm{P}$, ambos os capins apresentaram aumento linear na extração com aumento da CE na solução nutritiva. Essas observaçóes evidenciam que o capim-vetiver não é uma espécie considerada indicada para ser cultivada em SACsEHSS a serem utilizados no tratamento de águas residuárias ricas em nutrientes, devido à sua baixa capacidade de extraçáo. Entretanto, ao se comparar as extraçôes de nutrientes dos capins vetiver e tifton 85 , considerando-se dados obtidos para solução nutritiva de CE igual a $0,2 \mathrm{dS} \mathrm{m}^{-1}$, constata-se a superioridade do capim-vetiver, confirmando sua adaptação para cultivo em SACs-EHSS a serem utilizados no tratamento de águas residuárias contendo baixas concentraçóes de nutrientes.

Na Tabela 3, estão apresentados os parâmetros médios estimados dos polinômios de primeiro e segundo grau, ajustados de extração de N, P e K pelos capins vetiver e tifton 85 em função da CE na solução nutritiva, nas diferentes datas de corte das plantas, bem como os grupos formados pela análise de agrupamento por otimização de Tocher.

As estimativas da capacidade de extração de $\mathrm{N}$, $\mathrm{P}$ ou $\mathrm{K}$ pelo capim-vetiver pode ser realizada para as estaçóes de outono-inverno e primavera, a partir dos modelos comuns do grupo 1 e 2, respectivamente. Verifica-se que os modelos ajustados aos dados obtidos nos meses mais frios e, consequentemente, de menor produtividade de biomassa vegetal aérea, foram agrupados em um mesmo modelo, assim como, para os meses de maior produtividade, originando outro modelo comum.

A análise de agrupamento dos modelos de extração de $\mathrm{N}$ e $\mathrm{P}$ do capim-tifton 85 , em função da CE na solução nutritiva, possibilitou a obtenção de três grupos independentes. $\mathrm{O}$ modelo comum do grupo 1 representa os meses frios, em que a extração é baixa, já o modelo comum do grupo 3, caracteriza os meses mais quentes e de maior desenvolvimento vegetativo da planta, apesar de não ter sido a época de maior capacidade de extraçáo de $\mathrm{N}$ pelo referido capim. Já o modelo proveniente da data de avaliação no mês de setembro, pertencente ao grupo 2 , foi o que proporcionou a maior extração de $\mathrm{N}$ durante o período experimental, em consequência da produtividade de biomassa vegetal seca da parte aérea e do teor de $\mathrm{N}$ no tecido vegetal do capim-tifton 85 .

Os resultados das análises de agrupamento para extração de $\mathrm{K}$ pela parte aérea do capim-vetiver, assim como ocorreu em relação ao capim-tifton 85, resultaram em um grupo unitário para os dados obtidos no mês de novembro, consequência da interação produtividade de biomassa vegetal aérea e teor de K no tecido vegetal aéreo.

As condiçôes climáticas, principalmente a temperatura média do ar, apresenta elevada influência no desenvolvimento vegetativo dos capins, e deve ser considerado na avaliação da capacidade de extraçáo de nutrientes a partir dos modelos obtidos. Jesus (2016), observou que na estação de primavera houve maior remoção de nutrientes pelos capins vetiver e tifton quando comparado ao que foi obtido na estaçáo de inverno. Além disso, o correto manejo das plantas a partir de cortes frequentes nos períodos quentes do ano, potencializa o crescimento e evita o acamamento das plantas, maximizando a remoção de nutrientes nos SACs-EHSS.

\section{Validação dos modelos de estimativa da capacidade de extração de nutrientes}

$\mathrm{Na}$ Figura 2, estão apresentados os valores estimados, com intervalo de confiança de $95 \%$, da extração de N, $\mathrm{P}$ e $\mathrm{K}$ dos capins vetiver e tifton 85 para diferentes valores de CE no esgoto sanitário, assim como os valores reais obtidos nos SACs-EHSSp.

De acordo com os dados apresentados na Figura 2, a extração de $\mathrm{N}$ e $\mathrm{P}$ proporcionada pelo capim-vetiver cultivado no SAC-EHSSp, foi superior à estimativa realizada pelos modelos. Esses resultados se devem ao maior teor de $\mathrm{N}$ e $\mathrm{P}$ no tecido vegetal da parte aérea do capim-vetiver, uma vez que a produtividade em ambos os sistemas foi semelhante. Apesar da subestimativa, recomenda-se a utilização dos modelos do grupo 1 para estimar a extração de $\mathrm{N}$ e $\mathrm{P}$ na estação do inverno, considerada a mínima extração proporcionada pela planta. 
Tabela 3. Grupos formados e parâmetros médios estimados dos polinômios de primeiro e segundo grau $(\mathrm{f}(\mathrm{x})=$ $a+b x+c x 2$ ) ajustados de extração de N, P e K pelos capins vetiver e tifton 85 (g m-2 mês-1) em função da CE (dS m-1) na solução nutritiva saturante dos SACs-EHSS

\begin{tabular}{|c|c|c|c|c|c|c|c|}
\hline NUTRIENTE & CAPIM & GRUPO & DATA & $\mathrm{a}$ & $\mathrm{b}$ & c & $\mathrm{R}^{2}$ \\
\hline \multirow{18}{*}{ Nitrogênio } & \multirow{9}{*}{ Vetiver } & \multirow{6}{*}{1} & $16 / 05$ & $1,209^{* *}$ & $0,333^{*}$ & --- & 0,671 \\
\hline & & & $16 / 06$ & $0,799^{* *}$ & $0,362^{*}$ & --- & 0,789 \\
\hline & & & $16 / 07$ & $0,663^{*}$ & $0,618^{* *}$ & --- & 0,888 \\
\hline & & & $16 / 08$ & $0,815^{*}$ & $0,865^{* *}$ & --- & 0,859 \\
\hline & & & $16 / 09$ & $1,569^{*}$ & $1,540^{* *}$ & --- & 0,910 \\
\hline & & & M. Comum & $1,011^{*}$ & $0,743^{* *}$ & -- & 0,8848 \\
\hline & & \multirow{3}{*}{2} & $16 / 10$ & $2,570^{* *}$ & $1,295^{* *}$ & --- & 0,958 \\
\hline & & & $16 / 11$ & $4,094^{* *}$ & $1,390^{*}$ & -- & 0,831 \\
\hline & & & M. Comum & $3,332^{* *}$ & $1,343^{* *}$ & --- & 0,9588 \\
\hline & \multirow{9}{*}{ Tifton } & \multirow{4}{*}{1} & $16 / 05$ & $-1,497^{\text {ns }}$ & $9,301^{* *}$ & $-1,469^{*}$ & 0,989 \\
\hline & & & $16 / 06$ & $-0,586^{\mathrm{ns}}$ & $8,568^{*}$ & $-1,644 \bullet$ & 0,925 \\
\hline & & & $16 / 07$ & $-1,634^{\mathrm{ns}}$ & $11,223^{*}$ & $-2,193 \bullet$ & 0,949 \\
\hline & & & M. Comum & $-1,239^{\mathrm{ns}}$ & $9,697^{*}$ & $-1,769^{* *}$ & 0,982 \\
\hline & & 2 & $16 / 09$ & $-4,082^{*}$ & $25,004^{* *}$ & $-4,438^{* *}$ & 0,996 \\
\hline & & \multirow{4}{*}{3} & $16 / 08$ & $-2,221^{\mathrm{ns}}$ & $16,226^{* *}$ & $-3,225^{*}$ & 0,982 \\
\hline & & & $16 / 10$ & $-2,925^{*}$ & $19,926^{* *}$ & $-3,929^{* *}$ & 0,995 \\
\hline & & & $16 / 11$ & $-2,796^{\mathrm{ns}}$ & $18,172^{* *}$ & $-3,113^{*}$ & 0,989 \\
\hline & & & M. Comum & $-2,647^{* *}$ & $18,108^{* *}$ & $-3,423^{* *}$ & 0,999 \\
\hline \multirow{19}{*}{ Fósforo } & \multirow{9}{*}{ Vetiver } & \multirow{6}{*}{1} & $16 / 05$ & $0,153^{*}$ & $0,096^{*}$ & --- & 0,674 \\
\hline & & & $16 / 06$ & $0,090^{*}$ & $0,084^{* *}$ & --- & 0,917 \\
\hline & & & $16 / 07$ & $0,084^{*}$ & $0,099^{* *}$ & --- & 0,896 \\
\hline & & & $16 / 08$ & $0,099^{*}$ & $0,117^{* *}$ & --- & 0,934 \\
\hline & & & $16 / 09$ & $0,166^{*}$ & $0,209^{* *}$ & --- & 0,952 \\
\hline & & & M. Comum & $0,118^{*}$ & $0,121^{* *}$ & --- & 0,923 \\
\hline & & \multirow{3}{*}{2} & $16 / 10$ & $0,313^{* *}$ & $0,233^{* *}$ & --- & 0,986 \\
\hline & & & $16 / 11$ & $0,455^{*}$ & $0,374^{* *}$ & --- & 0,877 \\
\hline & & & M. Comum & $0,384^{* *}$ & $0,303^{* *}$ & --- & 0,937 \\
\hline & \multirow{10}{*}{ Tifton } & \multirow{4}{*}{1} & $16 / 05$ & $-0,044^{\mathrm{ns}}$ & $0,524^{* *}$ & --- & 0,995 \\
\hline & & & $16 / 06$ & $0,085^{\mathrm{ns}}$ & $0,453^{* *}$ & --- & 0,880 \\
\hline & & & $16 / 07$ & $0,030^{\mathrm{ns}}$ & $0,522^{* *}$ & --- & 0,951 \\
\hline & & & M. Comum & $0,024^{\mathrm{ns}}$ & $0,500^{* *}$ & --- & 0,965 \\
\hline & & \multirow{3}{*}{2} & $16 / 08$ & $0,100^{\text {ns }}$ & $0,702^{* *}$ & --- & 0,912 \\
\hline & & & $16 / 10$ & $0,219^{\text {ns }}$ & $0,800^{* *}$ & --- & 0,934 \\
\hline & & & M. Comum & $0,159^{\mathrm{ns}}$ & $0,751^{* *}$ & --- & 0,927 \\
\hline & & \multirow{3}{*}{3} & $16 / 09$ & $0,167^{\mathrm{ns}}$ & $1,010^{* *}$ & --- & 0,961 \\
\hline & & & $16 / 11$ & $0,284^{\mathrm{ns}}$ & $0,993^{* *}$ & --- & 0,878 \\
\hline & & & M. Comum & $0,225^{\mathrm{ns}}$ & $1,001^{* *}$ & --- & 0,934 \\
\hline & \multirow{5}{*}{ Vetiver } & \multirow{5}{*}{1} & $16 / 07$ & $0,529^{*}$ & $0,393^{*}$ & --- & 0,819 \\
\hline & & & $16 / 08$ & $0,758^{* *}$ & $0,390^{*}$ & --- & 0,796 \\
\hline & & & $16 / 09$ & $1,221^{*}$ & $0,869^{*}$ & --- & 0,822 \\
\hline & & & $16 / 10$ & $1,943^{* *}$ & $1,209^{* *}$ & --- & 0,921 \\
\hline Potássio & & & M. Comum & $1,113^{* *}$ & $0,715^{* *}$ & ..- & 0,8735 \\
\hline
\end{tabular}




\begin{tabular}{|c|c|c|c|c|c|c|}
\hline & 2 & $16 / 11$ & $5,573^{* *}$ & $1,668^{* *}$ & --- & 0,935 \\
\hline \multirow{8}{*}{ Tifton } & \multirow{7}{*}{1} & $16 / 05$ & $-0,461^{\mathrm{ns}}$ & $5,199^{* *}$ & $-0,642^{*}$ & 0,992 \\
\hline & & $16 / 06$ & $0,011^{\mathrm{ns}}$ & $4,191 \bullet$ & $-0,678^{\mathrm{ns}}$ & 0,914 \\
\hline & & $16 / 07$ & $-0663^{\text {ns }}$ & $5,093^{* *}$ & $-0,881^{*}$ & 0,989 \\
\hline & & $16 / 08$ & $-0,665^{\mathrm{ns}}$ & $7,058^{* *}$ & $-1,176^{*}$ & 0,976 \\
\hline & & $16 / 09$ & $-0,611^{\mathrm{ns}}$ & $10,428^{* *}$ & $-1,757^{*}$ & 0,984 \\
\hline & & $16 / 10$ & $-0,469^{\mathrm{ns}}$ & $9,226^{* *}$ & $-1,714^{*}$ & 0,983 \\
\hline & & M. Comum & $-0,476^{\mathrm{ns}}$ & $6,866^{* *}$ & $-1,141^{*}$ & 0,988 \\
\hline & 2 & $16 / 11$ & $-0,820^{\text {ns }}$ & $14,851^{* *}$ & $-3,159^{* *}$ & 0,988 \\
\hline
\end{tabular}

sendo: ** significativo em nível de 1\%; * significativo em nível de 5\%; •significativo em nível de 10\% e ns não significativo.
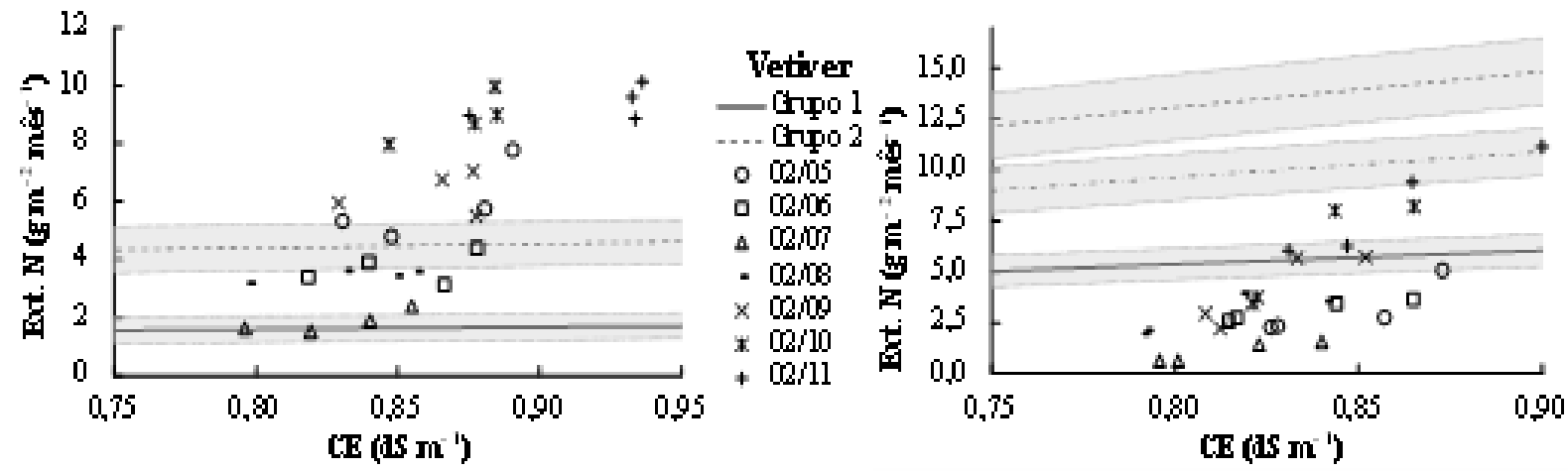

Tifton

Gruol

-. Grup 2

. Grumo

- 0205

口 0206

$\triangle 0207$

- 0208

$\times 02,09$

$\times 02 / 10$
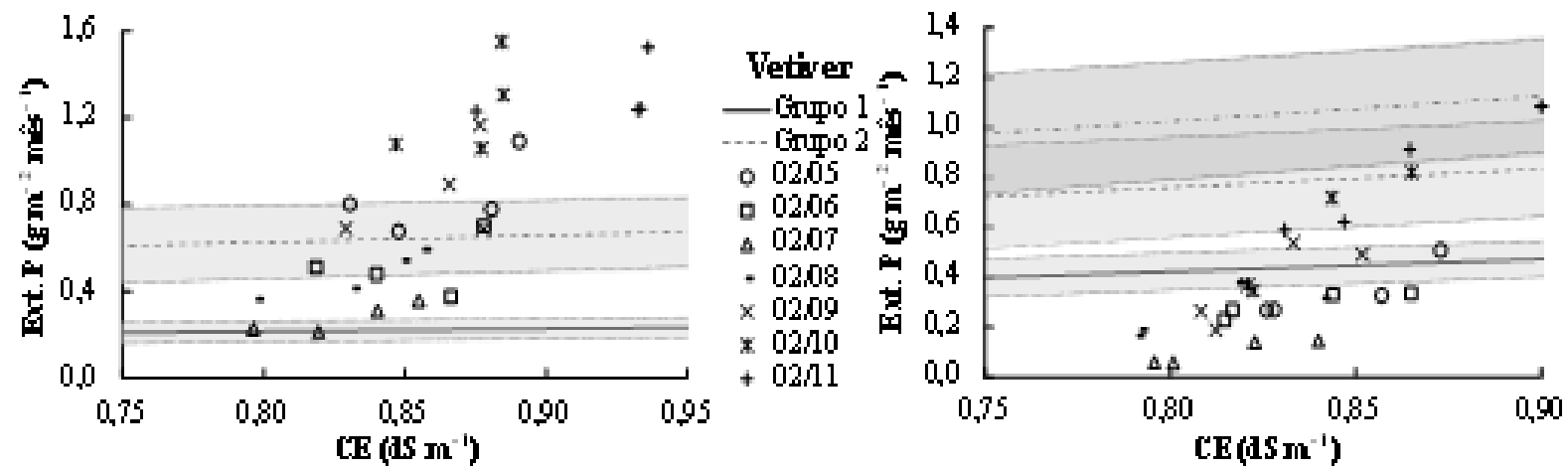

Tifton

- Grupo 1

-.... Gnqo 2

... Grupo 3

- 0205

- 0206

$\triangle 0207$

- 0208

$\times 0209$

$\times 0210$

CE ( $\left(\mathbb{S} \mathbf{m}^{-1}\right)$
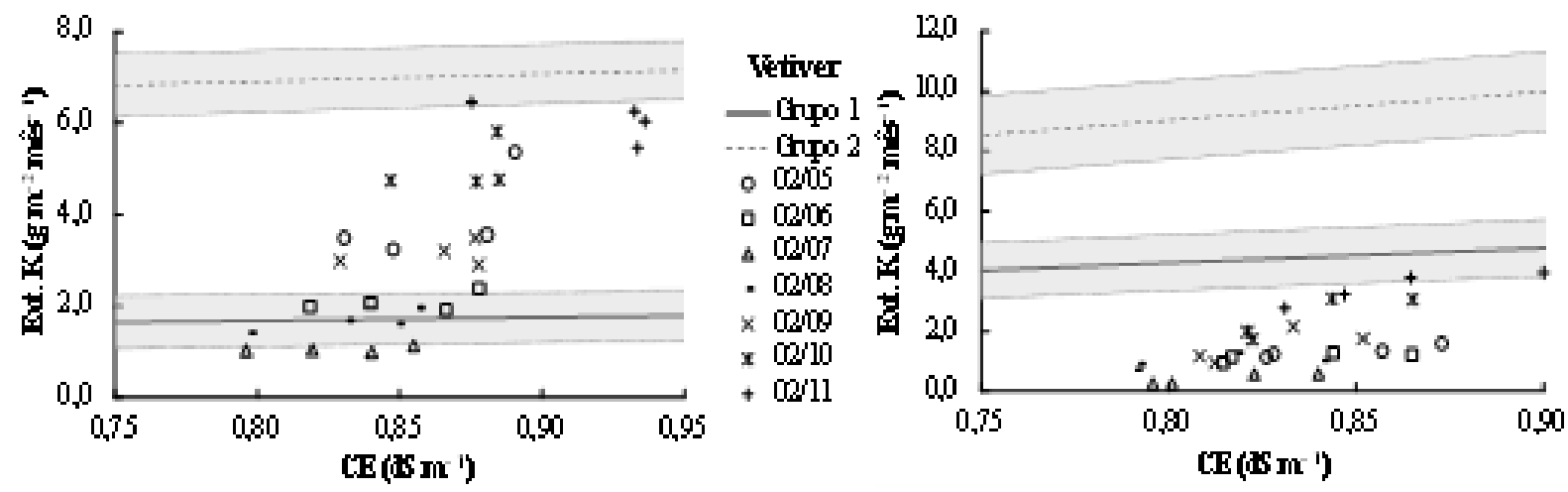

Tifion

- Gpol

.... Gupo 2

- 0005

- 00.06

\& 00.07

- 0208

$\times 000$

$\times 02 / 10$

+

Figura 2. Valores estimados e obtidos de extração de N, P e K dos capins vetiver e tifton 85, cultivados em SACsEHSSp, em função da CE no esgoto sanitário 
Os modelos de estimativa de extraçáo de $\mathrm{K}$ pela parte aérea do capim-vetiver possibilitaram estimativas satisfatórias dessa variável. $\mathrm{O}$ modelo do grupo 1 mostrou-se mais eficiente, pela maior proximidade dos valores obtidos no SAC-EHSSp, já o modelo do grupo 2 , proporcionou pequena superestimava nos valores de extração de K. Logo, a utilização do modelo do grupo 1 poderá fornecer uma faixa de valores esperados de extração de $\mathrm{K}$ via parte aérea do capim-vetiver cultivado em SACs-EHSSp utilizados no tratamento de esgoto sanitário.

Em relação ao capim-tifton 85 , os modelos de estimativa da extração de $\mathrm{N}, \mathrm{P}$ e K superestimaram os valores reais, consequência da maior produtividade de biomassa vegetal aérea nos SACs-EHSS. Acreditase que a menor extraçáo de nutrientes obtida no sistema real de tratamento esteja relacionada ao baixo desenvolvimento vegetativo do capim-tifton 85, o qual pode ser decorrente da idade das plantas e dos inúmeros cortes realizados em sua parte aérea. Segundo Carr et al. (1997), a idade das plantas e o processo de aclimatação ao ambiente que ocorre nas condiçóes reais de campo, pode influenciar na produtividade e proporcionar estimativas diferentes das obtidas, quando se utilizam dados provenientes de experimentos conduzidos sob condiçôes controladas.

Autores como Matos et al. $(2008,2009)$ e Fia et al. (2014) encontraram valores de extração de $\mathrm{N}$ e P pela parte aérea do capim-tifton 85 semelhantes aos observados nos protótipos de SACs-EHSS, por essa razão, recomenda-se a utilização dos modelos para estimativa da extraçáo de $\mathrm{N}$ e $\mathrm{P}$ pelo capim-tifton 85 , mesmo quando cultivado em SACs-EHSSp utilizados no tratamento de esgoto sanitário.

Apesar dos modelos gerados náo terem sidos validados de maneira satisfatória, esses podem ser considerados ferramentas essenciais para entender como as características da água residuária, a ser tratada em SACs-EHSS, podem afetar a capacidade extratora de nutrientes pelas plantas. Uma vez que, cada espécie apresenta limitada capacidade de remoção de nutrientes, resultando na estabilização ou até mesmo redução nas taxas de extração, com o aumento da carga de nutrientes (FIA et al., 2011, 2015; MENDONÇA et al., 2017).

\section{CONLUSÃO}

1. Modelos lineares de estimativa de extração de $\mathrm{N}, \mathrm{P}$ e $\mathrm{K}$, em sistemas alagados construídos, foram obtidos para o capim-vetiver. Em relação ao capimtifton 85 , foram ajustados modelos quadráticos de extração de $\mathrm{N}$ e $\mathrm{K}$, e lineares para a extração de $\mathrm{P}$.

2. Os modelos de estimativa da capacidade extratora de $\mathrm{N}$ e $\mathrm{P}$ pelo capim-vetiver, subestimaram a extração obtida nos SACs-EHSSp, sendo o modelo de estimativa de extração de K, validado com sucesso. Em relação ao capim-tifton 85 , os modelos superestimaram a capacidade extratora de N, P e K dos sistemas alagados construídos em escala plena.

3. Embora alguns modelos obtidos neste trabalho, não tenham sido validados de forma satisfatória, o conhecimento gerado contribui para o melhor aperfeiçoamento dos SACs-EHSS.

\section{LITERATURA CITADA}

APHA. Standard Methods for the Examination of Water and Wastewater, 22 ${ }^{\text {nd }}$ Edition. Washington: American Public Health Association, American Water Works Association, Water Environment Federation, 2012. 1496p.

CARR, G. M.; DUTHIE, H. C.; TAYLOR, W. D. Models of aquatic plant productivity: a review of the factors that influence growth. Aquatic Botany, v. 59, n. 3-4, p. 195-215, 1997.

DREIFUS, T. V. Tratamento de águas residuárias por alagados construídos de fluxo subsuperficial horizontal, utilizando vetiver (chrysopogon zizanoides): Avaliaçáo e desempenho de três leitos distintos. 2012. 84p. Dissertação (Mestrado em Engenharia Civil e Ambiental) - Universidade Estadual Paulista Júlio de Mesquita Filho, Bauru, SP, 2012.

FIA, F. R. L.; MATOS, A. T.; FIA, R.; BORGES, A. C.; ABREU, E. C. Influência da carga de nutrientes e da espécie cultivada na remoçáo de $\mathrm{K}, \mathrm{Na}, \mathrm{Cu}$ e $\mathrm{Zn}$ da água residuária da suinocultura tratada em sistemas alagados construídos. Ambiente \& Água-An Interdisciplinary Journal of Applied Science, v. 10, n. 3, p. 542-553, 2015. 
FIA, F. R. L.; MATOS, A. T.; FIA, R.; BORGES, A. C.; CECON, P. R. EFEITO DA VEGETAÇÃO EM SISTEMAS ALAGADOS CONSTRUÍDOS TRATANDO ÁGUAS RESIDUÁRIAS DA SUINOCULTURA. Engenharia Sanitária e Ambiental, v. 22, n. 2, p. 1-9, 2017.

FIA, F. R. L.; MATOS, A. T.; FIA, R.; LAMBERT, T. F.; PIMENTEL DE MATOS, M. Remoção de nutrientes por Typha latifolia e Cynodon spp. cultivadas em sistemas alagados construídos/Nutrients removal by Typha latifolia and Cynodon spp. grown in constructed wetlands. Revista Ambiente \& Água, v. 6 , n. 1, p. 77-89, 2011.

FIA, R.; BOAS, R. B. V.; CAMPOS, A. T.; FIA, F. R.; SOUZA, E. G. D. Removal of nitrogen, phosphorus, copper and zinc from swine breeding waste water by bermudagrass and cattail in constructed wetland systems. Engenharia Agrícola, v. 34, n. 1, p. 112-113, 2014.

GAO, J.; WANG, W.; GUO, X.; ZHU, S.; CHEN, S.; ZHANG, R. Nutrient removal capability and growth characteristics of Iris sibirica in subsurface vertical flow constructed wetlands in winter. Ecological Engineering, v. 70, p. 351-361, 2014.

HOAGLAND, D. R.; ARNON, D. I. The waterculture method for growing plants without soil. Circular - California Agricultural Experiment Station, v. 347, n. 2nd edit, 1950.

JESUS, F. L. F. Desempenho e influência dos capins tifton 85 (cynodon spp.) e vetiver (chrysopogon zizanioides) no tratamento de esgoto sanitário em sistemas alagados construídos. 2016. 93p. Dissertação (Mestrado em Engenharia Agrícola) Universidade Federal de Viçosa, Viçosa, MG, 2016.

KADLEC, R.H.; WALLACE, S. Treatment wetlands. CRC press, Boca Raton, Florida, 2008. 1016p.

KIEHL, E. J. Fertilizantes orgânicos. Piracicaba: Editora Agronômica Ceres, 1985. 492p.
MATOS, A. T. Manual de Análise de Resíduos Sólidos e Águas Residuárias. Viçosa: Editora UFV, 2015. 149p.

MATOS, A. T.; ABRAHÃO, S. S.; LO MONACO, P. A.; SARMENTO, A. P.; PIMENTEL DE MATOS, $M$. Capacidade extratora de plantas em sistemas alagados utilizados no tratamento de águas residuárias de laticínios. Revista Brasileira de Engenharia Agricola e Ambiental-Agriambi, v. 14, n. 12, p. 1311-1317, 2010 .

MATOS, A. T.; ABRAHÃO, S. S.; PEREIRA, O. G. Desempenho agronômico de capim tifton 85 (cynodon spp) cultivado em sistemas alagados construídos utilizados no tratamento de água residuária de laticínios. Ambiente \& Água-An Interdisciplinary Journal of Applied Science, v. 3, n. 1, p. 43-53, 2008.

MATOS, A. T.; FREITAS, W. S.; LO MONACO, P. A. V. Capacidade extratora de diferentes espécies vegetais cultivadas em sistemas alagados utilizados no tratamento de águas residuárias da suinocultura. Revista Ambiente \& Água, v. 4, n. 2, p. 31-45, 2009.

MENDONÇA, H. V.; MELO RIBEIRO, C. B.; NOGUEIRA, K. C. C. Remoção de matéria orgânica e nutrientes de águas residuais de laticínios em sistemas alagados construídos. Revista de Ciências Agrárias, v. 40, n. 1, p. 12-22, 2017.

MUSYIMI, D. M.; NETONDO, G. W.; OWUOR, B.; GICHIMU, B. M. Growth and nutrient uptake among three wetland plant species occurring in Lake Victoria basin in Kenya. Agriculture and biology journal of north america, v. 1, n. 3, p. 250-264, 2010.

UCKER, F. E.; ALMEIDA, R. A.; KEMERICH, P. D. C. Remoção de nitrogênio e fósforo do esgoto sanitário em um sistema de alagados construídos utilizando o capim vetiver. Ambiente \& Água-An Interdisciplinary Journal of Applied Science, v. 7, n. 3, p. 87-98, 2012. 